\title{
Predictive Evaluation of Quantitative Spatial Profiling of the Tumor Microenvironment by Multiplex Immunofluorescence in Recurrent Glioblastoma Treated with PD-1 Inhibitors
}

\section{David Alexander Cieremans ( $\nabla$ dcieremans@gmail.com )}

Columbia University Irving Medical Center https://orcid.org/0000-0002-1809-4733

Ju Young Kim

Navigate Biopharma

Ariana Valencia

Navigate Biopharma

Justin Santos

Navigate Biopharma

Jennifer Bordeaux

Navigate Biopharma

Thai Tran

Navigate Biopharma

Christine Vaupel

Navigate Biopharma

Naveen Dakappagari

Navigate Biopharma

Shabnam Tangri

Navigate Biopharma

Fabio Iwamoto

Columbia University Irving Medical Center

\section{Research Article}

Keywords: glioblastoma, immunotherapy, biomarkers, tumor microenvironment

Posted Date: June 1st, 2021

DOI: https://doi.org/10.21203/rs.3.rs-341251/v2

License: (9) This work is licensed under a Creative Commons Attribution 4.0 International License. Read Full License 
Version of Record: A version of this preprint was published at Journal of Clinical Oncology on May 20th, 2020. See the published version at https://doi.org/10.1200/JC0.2020.38.15_suppl.e14524. 


\section{Abstract}

Introduction: PD-1 inhibitors have shown limited efficacy in glioblastoma due to microenvironment immunosuppression and low tumor mutational burden. In GBM, PD-L1 expression is not a predictive marker for response to PD-1 or PD-L1 inhibitors. Multiplex immunostaining panel technology allows for detailed analyses of tumor microenvironment cells and their interaction. Our study to evaluates the feasibility and predictive value of quantitative spatial profiling in GBM.

Methods: Pre-treatment tumor tissue was collected retrospectively from 27 patients at Columbia University Irving Medical Center with primary glioblastoma who were diagnosed within the past three years, had surgery here, and were either treated with SOC therapy $(n=8)$ or PD-1 inhibitors at recurrence $(\mathrm{n}=19)$. Multiplex immunofluorescence panels included 1) CD11b/ID01/HLADR/GFAP, 2) PD1/PDL1/GFAP, and 3) CD4/CD8/CD25/FoxP3/Ki67/GFAP.

Results: Multiplex immunofluorescence panels did not show any correlation with outcomes in patients treated with SOC therapy. Among the 19 patients treated with PD-1 inhibitors, those with more HLA-DR positive cells had worse outcomes $(p=0.02)$. PD-L1 expression on tumor cells was not predictive of outcomes. There was a correlation trend between PD-1/PD-L1 interaction score $(p=0.08)$ and outcomes. PTEN loss was correlated with higher Ki67 expression in both tumor cells $(p=0.05)$ and non-tumors cells $(p=0.03)$; however, this was not found in Ki67 in CD4+ cells, CD8+ cells, or CD4+CD8+ cells combined. Tumor-associated macrophages, myeloid-derived suppressor cells, CD8+ cells, and CD4+ cells were not significant predictive markers for outcome.

Conclusion: Quantitative spatial profiling by multiplex immunofluorescence is feasible in FFPE glioblastoma tissue. More refined and extensive quantitative and spatial microenvironment analyses may allow for the development of biomarkers for immunotherapy in GBM.

\section{Introduction}

Glioblastoma (GBM) is the most aggressive primary brain tumor (glioma) in adults (WHO Grade IV) and accounts for more than half of all brain tumors [1]. Even after surgery, chemotherapy, and radiation therapy, tumors often recur and survival remains poor at fewer than 15 months after diagnosis [2]. Common pathological biomarkers include methylation (MGMT) status, isocitrate dehydrogenase (IDH) mutation, epidermal growth factor receptor (EGFR) amplification, and phosphatase and tensing homolog (PTEN), all of which have prognostic relevance or may be predictors of response to specific therapy. PTEN is a tumor suppressor gene that plays an integral role in cell proliferation regulation, adhesion and invasion, apoptosis, and DNA damage repair. PTEN loss in melanoma tumor cells increases expression of immunosuppressive cytokines, which leads to decreased T cell infiltration in tumors, and inhibition of autophagy, which regulates T cell-mediated cell death [3]. Loss of PTEN expression begins early in glioma development with mutations occurring in between $5 \%$ and $40 \%$ of cases [4]. Zhao et al. demonstrate in their cohort of GBM patients treated with immunotherapy that non-responders had tumors enriched in 
PTEN mutations. RNA-sequencing has also indicated that the PTEN mutation may induce an immunosuppressive microenvironment and lead to shorter overall survival. As a result, PTEN status has become a valuable biomarker in the investigation of the tumor microenvironment in GBM.

Standard of care (SOC) therapy typically includes maximal surgical resection followed by radiation therapy and concomitant and adjuvant temozolomide. Better treatment strategies are urgently needed and immunotherapies such as programmed cell death protein-1 (PD-1) inhibitors are in clinical trials for the treatment of GBM. Immune checkpoint inhibitors of PD-1 have revolutionized the treatment of other cancer types, such as lung cancer and melanoma, by blocking PD-1 (on effector T cells) which interacts with PD-L1 on the surface of cancer cells to suppress immune function. Programmed death-ligand 1 (PDL1) testing helps identify patients who may benefit from anti-PD-1 therapy in many tumor types. In GBM specifically, PD-L1 is not a predictive marker for response to PD-1 or PDL-1 checkpoint inhibitors, due primarily to the variance in expression and subcellular distribution of PD-L1 in glioma cells [5]. A large phase 3 study using the PD-1 inhibitor nivolumab in recurrent glioblastoma (Checkmate 143 from BMS) showed an $8 \%$ overall response rate in GBM but no predictive biomarker of response [6]. Finding a biomarker that can predict response to PD-1 inhibitors would be extremely helpful in GBM as this would allow for the selection of patients most likely to benefit from such therapy.

The lower efficacy of PD-1 inhibitors in GBM may be due to microenvironment immunosuppression and low tumor mutational burden (TMB). The higher the number of mutations per coding area of a tumor genome yields a more stimulated immune response and more favorable tumor response to immunotherapy. Hodges et al. discovered that high TMB was found in only $3.5 \%$ of GBM patients and that both high and moderate TMB GBMs did not have an enriched influx of CD8+ T cells, PD-1+ T-cells, or tumor-expressed PD-L1 cells [7]. They also demonstrated that the subset of GBMs that should have a better response to immune checkpoint inhibitors based on TMB still lacked the typical biomarkers of response, further suggesting the limited significance of PD-1 and PD-L1 testing in GBM. Nevertheless, finding a more specific and accurate testing of these biomarkers could be beneficial in treating certain GBMs due to the severe lack of proven treatment options.

Multiplex immunostaining panel technology has explored novel biomarkers in melanoma and identified two which have prognostic significance in overall survival, namely PD-1/PD-L1 interaction score, which measures density PD-1-positive cells in proximity to PD-L1-positive cells in contrast to measuring PD-L1 alone, and colocalization of human leukocyte antigen-DR (HLA-DR, $D=$ aspartic acid, $R=$ arginine) and indoleamine-2,3-dioxygenase-1 (IDO-1), which are upregulated by interferon gamma (IFNy) in tumors rich with immune cells [8]. Our primary hypothesis was that although response to PD-1 immunotherapy in GBM is low, multiplex immunostaining panel technology is feasible on GBM tumor tissue and will allow for detailed analysis of tumor microenvironment cells and their interaction with one another. Our secondary hypothesis was that PD-1 immunotherapy based on PD-1/PD-L1 interaction score and IDO/HLA-DR expression can predict overall survival (OS) and survival from initiation of PD-1 therapy. 
HLA-DR is an MHC class II cell surface receptor that presents peptide antigens to the immune system to mediate $T$ cell response [9]. IDO-1 is a tryptophan-metabolizing enzyme that regulates immunologic tolerance and whose expression varies across tumor types [10]. IFNy is a cytokine essential to innate and adaptive immunity, particularly in the activation of macrophages and inducer of class II MHC molecule expression [11]; it acts to limit tumor growth and induces IDO and HLA-DR expression on tumor cells. Johnson et al. demonstrated that high PD-1/PD-L1 interaction and high ID0-1/HLA-DR were statistically significant different between responders and non-responders; however, coexpression of both biomarkers had the best response to anti-PD-1 therapy and was correlated with the best survival outcome [8]. Unfortunately, tumor cells and T cells in the microenvironment of GBM produce low levels of IFNy, thereby decreasing tumor immunogenicity and disrupting regulatory mechanisms to curtail proliferation and angiogenesis [12]. Like PD-L1, IDO-1/HLA-DR levels could also provide insight into potential response to checkpoint inhibitor immunotherapy in GBM.

More generally, this technology provided us a comprehensive snapshot of the microenvironment of GBM. Myeloid-derived suppressor cells (MDSCs) represent a heterogeneous population of myeloid cells that are present in cancer, inflammation, and infection [13]. The inhibitory effects of MDSCs on innate and adaptive immunity lead to blocking immune surveillance and preventing the immune system from eliminating newly transformed cells. MDSCs can also differentiate into tumor-associated macrophages (TAMs) within the tumor environment. TAMs promote tumor growth, regulate metastasis, mediate response to therapy, and often lead to worse survival outcome. The expression of specific cell surface markers such as CD11b (a marker of macrophages and microglia which regulates leukocyte adhesion and migration to mediate inflammatory response), HLA-DR, and MDSC-specific enzymes such as IDO-1 makes it possible to identify MDSCs [14].

T cells also play a critical role in the immune response and are affected by MDSCs. They can be separated into three major groups based on function: cytotoxic T cells (CD8+), helper T cells (CD4+), and regulatory $T$ cells ( $T$ regs). Differential expression of markers on the cell surface, as well as their distinct cytokine secretion profiles, provide valuable clues to the diverse nature and function of $T$ cells. Immunohistochemistry can help to classify a subset of $T$ cells and their activation status by the presence of Ki67, a marker for proliferation, and FoxP3, a marker for immunosuppression through $\mathrm{T}$ cell regulation. Many primary tumors have massive infiltration of macrophages, which can make up 30-50\% of tumor mass including central nervous system (CNS) tumors like GBM and brain metastases [15]. In general, counts of low CD8+ cells, high CD4+ cells, high T regulatory cells, low IFNy, and high TAMs correlate with a more immunosuppressed microenvironment. The brain uses two types of cells in the immune response: microglia as the first line of defense and macrophages recruited from the tumor periphery or outside the brain. Chen et al. demonstrate that the macrophage marker CD45Hi accounts for $1.5(+/-3) \%$ of total brain myeloid cell population in naïve mice and $87.5(+/-6.2) \%$ in mice with brain tumors [16]; however, $\mathrm{CD} 45 \mathrm{Hi}$ levels can only differentiate macrophages and microglia under steady-state conditions and not under inflammatory conditions. This suggests the integral role of macrophage recruitment in the localized tumor immunosuppression in gliomas and provide a framework for investigating other biomarkers that may respond to targeted immunotherapy intervention. 


\section{Materials And Methods}

We evaluated the density of PD-1/PD-L1 and ID0-1/HLA-DR and counts of CD8+ cells, CD4+ cells, T regulatory cells, TAMs, and myeloid derived suppressor cells in patients with recurrent glioblastoma who have been treated with PD-1 checkpoint inhibitors (nivolumab or pembrolizumab) for predictive significance. We used tumor samples from patients with recurrent GBM $(n=27)$ treated with either SOC therapy (RT/TMZ/etc.) $(n=8)$ or single-agent anti-PD-1 therapy (pembrolizumab or nivolumab) $(n=19)$ at Columbia University Irving Medical Center after 2017. Most common SOC therapies at recurrence included bevacizumab, lomustine, and optune. We obtained all retrospective specimens from formalinfixed, paraffin-embedded (FFPE) biopsy or resection tissue from our neurooncology database at new diagnosis of GBM prior to initiation of SOC or PD-1 therapy at recurrence. Participation in the neurooncology database allowed us to collect age, gender, ethnicity, risk factors for brain or spinal tumors, seizures, surgery date, clinical test results such as MRI findings, pathological diagnosis, radiation therapy, chemotherapy, use of medications, participation in clinical trials, patient-derived data, time to progression and survival time, and tumor tissue for use in our study. We submitted these samples to Navigate Biopharma for quantitative immunofluorescence (QIF) (Fig. 1-3) and evaluation using automated quantitative analysis (AQUA) technology. We then performed statistical analysis on the cell counts to correlate individual biomarker variables with one another and survival outcomes. These tests have more biological relevance for the PD-1 treated patients than those on SOC therapy.

\section{Immunofluorescence staining, sample imaging, and imaging analyses by AQUA technology (8)}

\section{See Johnson et al. for laboratory protocol. Methods adapted for GBM specific testing.}

\section{Statistical analysis}

The primary analytic framework utilized the Kaplan-Meier method, Cox proportional hazards models of time to events, and logistic regressions using baseline characteristic data as well as the multiplex immunofluorescence data. Overall survival (OS) from initial diagnosis in PD-1 and SOC groups and survival from PD-1 dosing start were also assessed. The p-values, hazard ratios, and confidence intervals for correlation between each variable and OS were determined using Cox proportional hazard regression models. Similarly, p-values, odds ratios, and confidence intervals were generated using logistic regression to evaluate the potential correlation between PTEN status as an indicator of tumor immunosuppression and biomarkers under study. Baseline characteristics such as median age, MGMT status, IDH status, PTEN status, Karnofsky Performance Status (KPS) at recurrence (which measures functional impairment), median lines of therapy before PD-1, median time from diagnosis to PD-1 (months), median time on PD-1 (months) were also measured and compared (Table 1). Because we focused solely on recurrent GBM, PD-1 markers were more significant than either IDH or MGMT status. All analyses were performed using Excel and Stata. 


\section{Results}

\section{PD-1/PD-L1 density score has correlation trend with overall survival in GBM, but HLA-DR does not}

Quantitative spatial profiling of the tumor microenvironment by multiplex immunofluorescence allowed us to evaluate tumor-associated macrophages, myeloid-derived suppressor cells, CD8+ lymphocytes, and CD4+ $\mathrm{T}$ regulatory cells in recurrent GBM for prognostic significance; however, none of these were significant predictive markers for survival. Because the biomarkers under study are only relevant to immunotherapy treatment response, these panels did not show any correlation with outcomes in patients treated with SOC therapy. Besides PD-1/PD-L1 interaction score and HLA-DR levels in all cells, no other biomarker was correlated with overall survival. Johnson et al. previously demonstrated that PD-1 and PDL1 need to be expressed in close proximity and in sufficient quantity to demonstrate response to PD-1 inhibitor therapy [8]. We tested this hypothesis and investigated the validity of these results in our patient population. Although not statistically significant, the PD-1/PD-L1 interaction score had a correlation trend with survival $(p=0.08, \mathrm{HR}=1.05,95 \% \mathrm{Cl}=0.99,1.11)$ but further research is needed to validate potential clinical relevance. Although still routinely tested in pathology, PD-L1 expression on tumor cells is not predictive of outcome; we confirmed this in our subset of patients as well and found no correlation with survival $(p=0.97, \mathrm{HR}=0.99,95 \% \mathrm{Cl}=0.97,1.03)$. We used Cox proportional hazard models to evaluate correlation between biomarkers and OS. Unlike Johnson et al., where in melanoma the combination of high PD-1/PD-L1 interaction score and/or ID0-1/HLA-DR positivity demonstrated best response, we found that patients with more positive HLA-DR cells had worse OS $(p=0.02, \mathrm{HR} 1.05,95 \% \mathrm{Cl}=1.00,1.09)$.

\section{PTEN loss/Ki67+ coexpression as marker of GBM tumor microenvironment}

Because PTEN mutation is a marker of the tumor microenvironment immunosuppression in GBM, we performed logistical regression between PTEN status and the remaining biomarkers under study to investigate any potential correlations. We identified in our subset of patients that PTEN loss was correlated with higher Ki67 expression in both tumor cells ( $p=0.05, \mathrm{OR}=1.61,95 \% \mathrm{Cl}=1.01,2.57)$ and non-tumors cells $(p=0.03, O R=1.38,, 95 \% C l=1.03,1.84)$; however, this relationship was not found when looking at Ki67 in CD4+ cells, CD8+ cells, or CD4+CD8+ cells combined. These findings indicate how PTEN loss/Ki67+ coexpression may have biological plausibility in GBM and offer predictive relevance, like PD-1/PD-L1 density score and HLA-DR/IDO-1 coexpression in response to immunotherapy.

\section{Discussion}

In this study, we demonstrated the efficacy and feasibility of quantitative spatial profiling by multiplex immunofluorescence in investigating the tumor microenvironment cells of GBM and their interactions with one another. We also found that a) PD-1/PD-L1 interaction score may be a predictor of OS in recurrent GBM patients who received anti-PD-1 therapy, b) HLA-DR is associated with worse OS in recurrent GBM patients who received anti-PD-1 therapy, and c) the coexpression of PTEN loss and Ki67+ 
tumor and non-tumor cells may provide a more specific marker of immunosuppression in the GBM microenvironment.

HLA-DR is a generic, non-specific biomarker in GBM; however, Fan et al. prove that an increased HLA-DR score was correlated with a more aggressive glioma tumor grade [17]. Additionally, they investigated whether HLA-DR scores predicted survival in GBM patients treated with immunotherapy but found no significant difference in the prognosis of subgroups with high and low HLA-DR scores. Diao et al. found that GBM patients with high content of HLA-DR had a survival rate of $16.7 \%(6 / 36)$ compared to patients with low content of HLA-DR at 38.9\% (7/18) [18]. Although only 41 patients treated with SOC therapy were included, this study mirrored our findings. As discussed, myeloid cells can present HLA-DR and differentiate into TAMs, which correlate with an immunosuppressed tumor microenvironment, low tumor mutational burden, and low response; this is in contrast to melanoma which had a higher response with more HLA-DR+ cells. Therefore, GBM with higher levels of HLA-DR+ cells may not respond well to either SOC or immunotherapy.

We compared our findings of PTEN loss and Ki67+ coexpression against those from previous studies. Ji et al. studied the relationship between PTEN and Ki67 expression in non-small cell lung cancer and found that PTEN mutation was correlated with higher Ki67 expression in tumor cells as opposed to normal lung tissue [19]. Additionally, they determined that low PTEN expression and high Ki67 expression are associated with high proliferation activity, low differentiation of tumor tissues, and high potential for early invasion and metastasis. Zhao et al. studied the transcriptomal profiles from three GBMs, including a PTEN-mutated tumor [3]. They found that prior to immunotherapy, tumor cells clustered more strongly with each other in PTEN-mutated cases compared to PTEN-wild-type $(\mathrm{p}=2.4 \mathrm{e}-4)$. The upregulation of these immune signatures in non-responsive tumors suggests the role of PTEN mutation and Ki67 in the development of the tumor microenvironment, further validating our findings. Zhao et al. also discuss how FoxP3-expressing $T$ regulatory cells suppress aberrant immune response against self-antigens and are negatively associated with clinical response to adaptive immunotherapy in human cancers; they found that PTEN mutations are significantly correlated with the FoxP3 $(\mathrm{p}=0.028)$, although we did not see this in our selected-study population. Because our sample size was small and we performed many logistical regressions at $p=0.05$, we must be skeptical of our results. These findings will need to confirmed with a larger number of patients in future studies using the same multiplex immunofluorescence technology.

The strengths of this analysis include its objective and quantitative nature, minimal tissue requirements ( 2 unstained slides), high predictive capacity, and potential for use in other combination assays. The higher predictive value of these assays can be attributed to the unique operating principles of the underlying digital pathology approach, which uses spatial information and signal enrichment of rare cellular phenotypes within the tumor microenvironment. Due to the limited number of patients in our study, more refined and extensive quantitative and spatial microenvironment analyses may allow for the development and confirmation of biomarkers for immunotherapy in GBM. Future mFIHC panels will instead use CD8/GZMB/PD1/PDL1/TIM3/GFAP, CD3/CD11b/CD19/HLADR/ID01/GFAP, and CD3/CD4/CD8/FOXP3/KI67/GFAP panels. Advantages include more biomarker information, spatial 
information across the entire tissue, and fully-automated staining using Leica Bond RX for improved reproducibility. To validate our initial findings and examine the value of this new assay, our next cohort will have approximately 100 patients split between discovery and validation cohorts. The discovery cohort will be separated into $50 \%$ responder and $50 \%$ non-responder, and the evaluated signature from discovery cohort will be used in validation cohort.

In conclusion, our study identified multiple biomarkers related to anti-PD-1 therapy in GBM and investigated the potential associations between survival outcomes and the biomarkers themselves. Though limited by sample size, we have shown through our statistical analysis both the significance and promise of this assay in detailing the tumor microenvironment of GBM. Like Zhao et al., who also reported that their patients might benefit from anti-PD-1 inhibitors based on extensive genomic and spatial testing, we are confident that a highly-specific analysis utilizing quantitative spatial profiling by multiplex immunofluorescence can provide predictive value in selecting GBM patients for treatment with PD-1 immunotherapy.

\section{List Of Abbreviations}

GBM: glioblastoma

WHO: World Health Organization

CNS: central nervous system

MGMT: 06-methylguanine-DNA-methyltransferase

PTEN: phosphatase and tensin homolog

IDH: isocitrate dehydrogenase

WT: wild type

MUT: mutant

EGFR: endothelial growth factor receptor

SOC: standard of care

TMZ: temozolomide

FFPE: formalin-fixed, paraffin-embedded

PD-1: programmed cell death protein-1

PD-L1: programmed death-ligand 1 
ID0-1: indoleamine-2,3-dioxygenase-1

HLA-DR: human leukocyte antigen-DR

D: aspartic acid

R: arginine

IFNy: interferon gamma

TAMs: tumor-associated macrophages

TMB: tumor mutational burden

CD11b: tumor-infiltrating myeloid cells

GFAP: glial fibrillary acidic protein

CD4: cluster of differentiation 4

CD8: cluster of differentiation 8

CD25: interleukin-2 receptor alpha chain

FoxP3: forkhead box protein 3

Ki67: antigen Ki67

KPS: Karnofsky Performance Status

OS: overall survival

\section{Declarations}

Funding: Navigate BioPharma Services, Inc., a Novartis subsidiary

Conflicts of Interest/competing interests: Not applicable

Availability of data and material: manuscript data included for public review

Code availability: Not applicable

Authors' contributions: Not applicable

Ethics approval: Not applicable

Consent to participate: Subjects consented to participation 
Consent for publication: Authors consented to publication

\section{References}

1. Hanif F, Muzaffar K, Perveen K, Malhi SM, Simjee ShU (2017) Glioblastoma Multiforme: A Review of its Epidemiology and Pathogenesis through Clinical Presentation and Treatment. Asian Pac J Cancer Prev 18(1):3-9. doi: 10.22034/APJCP.2017.18.1.3.

2. Davis ME (2016) Glioblastoma: Overview of Disease and Treatment. Clin J Oncol Nurs 20(5): S2-S8. doi: 1188/16.CJON.S1.2-8.

3. Zhao J, Chen AX, Gartrell RD, Silverman AM, Aparicio L, Chu T, Bordbar D, Shan D, Samanamud J, Mahajan A, et al. (2019) Immune and genomic correlates of response to anti-PD-1 immunotherapy in glioblastoma. Nat Med 25(3):462-469. doi: 10.1038/s41591-019-0349-y.

4. Han F, Hu R, Yang H, Liu J, Sui J, Xiang X, Wang F, Chu L, Song S (2016) PTEN gene mutations correlate to poor prognosis in glioma patients: a meta-analysis. Onco Targets Ther 9:3485-92. doi: 10.2147/OTT.S99942.

5. Chen RQ, Liu F, Qiu XY, Chen XQ (2019) The Prognostic and Therapeutic Value of PD-L1 in Glioma. Front Pharmacol 9:1503. doi: 10.3389/fphar.2018.01503.

6. Reardon DA, Omuro A, Brandes A, Rieger J, Wick A, Sepulveda J, Phuphanich S, de Souza P, Ahluwalia MS, Lim M, et al. (2017) Randomized Phase 3 Study Evaluating the Efficacy and Safetyof Nivolumab vs Bevacizumab in Patients With Recurrent Glioblastoma: CheckMate 143. Neuro Oncology 19(3):iii21. org/10.1093/neuonc/nox036.071.

7. Hodges TR, Ott M, Xiu J, Gatalica Z, Swensen J, Zhou S, Huse JT, de Groot J, Li S, Overwijk WW, et al. (2017) Mutational burden, immune checkpoint expression, and mismatch repair in glioma: implications for immune checkpoint immunotherapy. Neuro Oncology 19(8):1047-1057. doi: 10.1093/neuonc/nox026.

8. Johnson DB, Bordeaux J, Kim JY, Vaupel C, Rimm D, Ho TH, Joseph RW, Daud Al, Conry RM, Gaughan EM, et al. (2018) Quantitative Spatial Profiling of PD-1/PD-L1 Interaction and HLA-DR/IDO1 Predicts Improved Outcomes of anti-PD-1 Therapies in Metastatic Melanoma. Clin Cancer Res 34(21): 5250-5260. doi: 1158/1078-0432.CCR-18-0309.

9. Janeway CA, Travers P, Walport M, Shlomchik M. (2001) Immunobiology, $5^{\text {th }}$ Edition: The Immune System in Health and Disease. Garland Science.

10. Munn D, Mellor A (2017) IDO in the Tumor Microenvironment: Inflammation, Counter-regulation and Tolerance. Trends Immunol 37(3): 193-207. doi: 1016/j.it.2016.01.002.

11. Tau G, Rothman P (1999) Biologic functions of the IFN-y receptors. Allergy 54(12): 1233-1251. doi:10.1034/j.1398-9995.

12. Castro F, Cardoso AP, Goncalves RM, Serre K, and Oliveira MJ (2018) Interferon-Gamma at the Crossroads of Tumor Immune Surveillance or Evasion. Front Immunol 9: 847. doi: 3389/fimmu.2018.00847. 
13. Gieryng A, Kaminska B (2016) Myeloid-derived suppressor cells in gliomas. Contemp Oncol (Pozn) 20(5):345-351. doi: 10.5114/wo.2016.64592.

14. Novus Biologicals (2011) CD11b Expression, Leukocyte Adhesion and the Innate Immune System. Internet: https://www.novusbio.com/antibody-news/antibodies/cd11b-expression-and-the-innateimmune-system (accessed 01 April 2020).

15. Bingle L, Brown NJ, Lewis CE (2002) The role of tumour-associated macrophages in tumour progression: implication for new anticancer therapies. J Pathol 196(3):254-65. doi:1002/path.1027.

16. Zhihong C, Feng X, Hertin CJ, Garcia VA, Nie K, Pong WW, Rasmussen R, Dwivedi B, Seby S, Wolf SA, et al. (2017) Cellular and Molecular Identity of Tumor-Associated Macrophages in Glioblastoma. Cancer Res 77(9):2266-2278. doi:1158/0008-5472.CAN-16-2310.

17. Fan X, Liang J, Wu Z, Shan X, Qiao H, Jiang T (2017) Expression of HLA-DR genes in gliomas: correlation with clinicopathological features and prognosis. Chin Neurosurg JI3(27). doi:1158/10780432.CCR-18-0309.

18. Diao J, Xia T, Zhao H, Liu J, Li B, Zhang Z (2015) Overexpression of HLA-DR is associated with prognosis of glioma patients. Int J Clin Exp Pathol 8(5):5485-90.

19. Ji Y, Zheng M, Ye S, Chen J, Chen Y (2014) PTEN and Ki67 expression is associated with clinicopathologic features of non-small cell lung cancer. J Biomed Res 28(6):462-7. doi: 10.7555/JBR.27.20130084.

\section{Tables}

Table 1- Baseline characteristics of PD-1 and SOC treated groups. Number of patients per characteristic is listed in parentheses. (MGMT: 06-methylguanine-DNA-methyltransferase (methylation), IDH: isocitrate dehydrogenase, WT: wild type, MUT: mutant, PTEN: phosphatase and tensin homolog, KPS: Karnofsky Performance Status, RT: radiation therapy, TMZ: temozolomide, OS: overall survival) 


\begin{tabular}{|c|c|c|}
\hline Baseline Characteristics & cases $(n=19)$ & controls $(n=8)$ \\
\hline median age (years) & 55. & $.2 \quad 58.8$ \\
\hline \multicolumn{3}{|l|}{ MGMT status (\%) } \\
\hline methylated & $21(4)$ & $12.5(1)$ \\
\hline unmethylated & $68.4(13)$ & $75(6)$ \\
\hline UNK & $10.5(2)$ & $12.5(1)$ \\
\hline \multicolumn{3}{|l|}{ IDH status (\%) } \\
\hline IDH WT & $78.9(15)$ & $12.5(1)$ \\
\hline IDH MUT & $15.7(3)$ & $75(6)$ \\
\hline variable/UNK & $12.5(1)$ & $12.5(1)$ \\
\hline \multicolumn{3}{|l|}{ PTEN status (\%) } \\
\hline PTEN negative & $36.8(7)$ & $25(2)$ \\
\hline PTEN positive & $57.9(11)$ & $75(6)$ \\
\hline UNK & $5.2(1)$ & $0(0)$ \\
\hline KPS & $80(19)$ & $80(7)$ \\
\hline received RT/TMZ (\%) & $100(19)$ & $100(8)$ \\
\hline median lines therapy before PD-1 & $3(19)$ & N/A \\
\hline median time from diagnosis to PD-1 (months) & $14.24(19)$ & N/A \\
\hline median time on PD-1 (months) & $10.76(19)$ & N/A \\
\hline median survival from PD-1 start (months) & $13.95(19)$ & N/A \\
\hline OS (months) & $39.1(19)$ & $19.1(8)$ \\
\hline
\end{tabular}

\section{Figures}

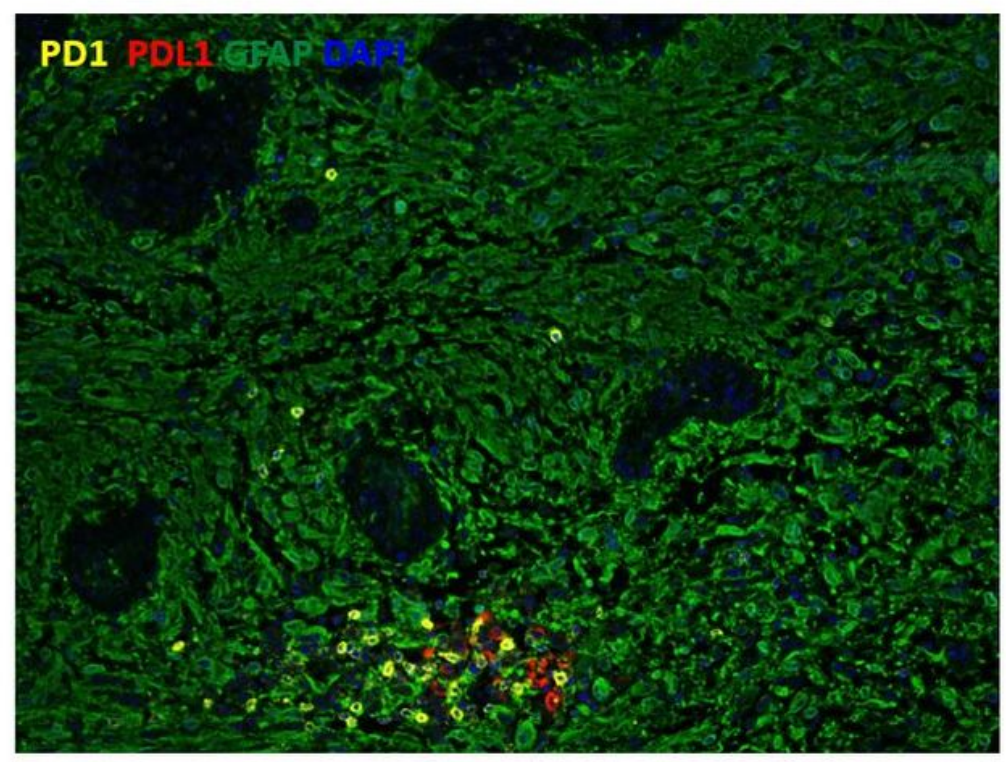

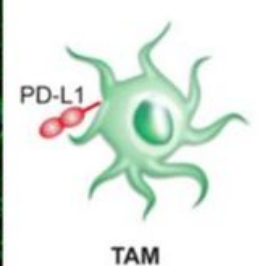
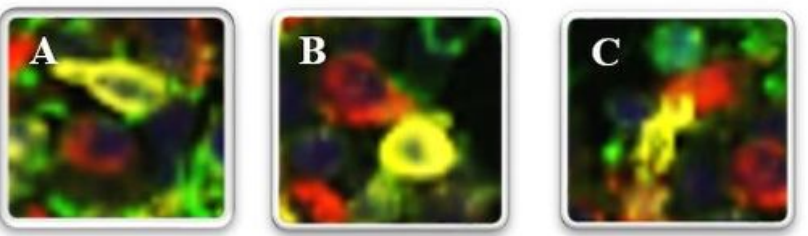

Figure 1

Individual PD-1/PD-L1 interactions in GBM tissue (A, B, C) 

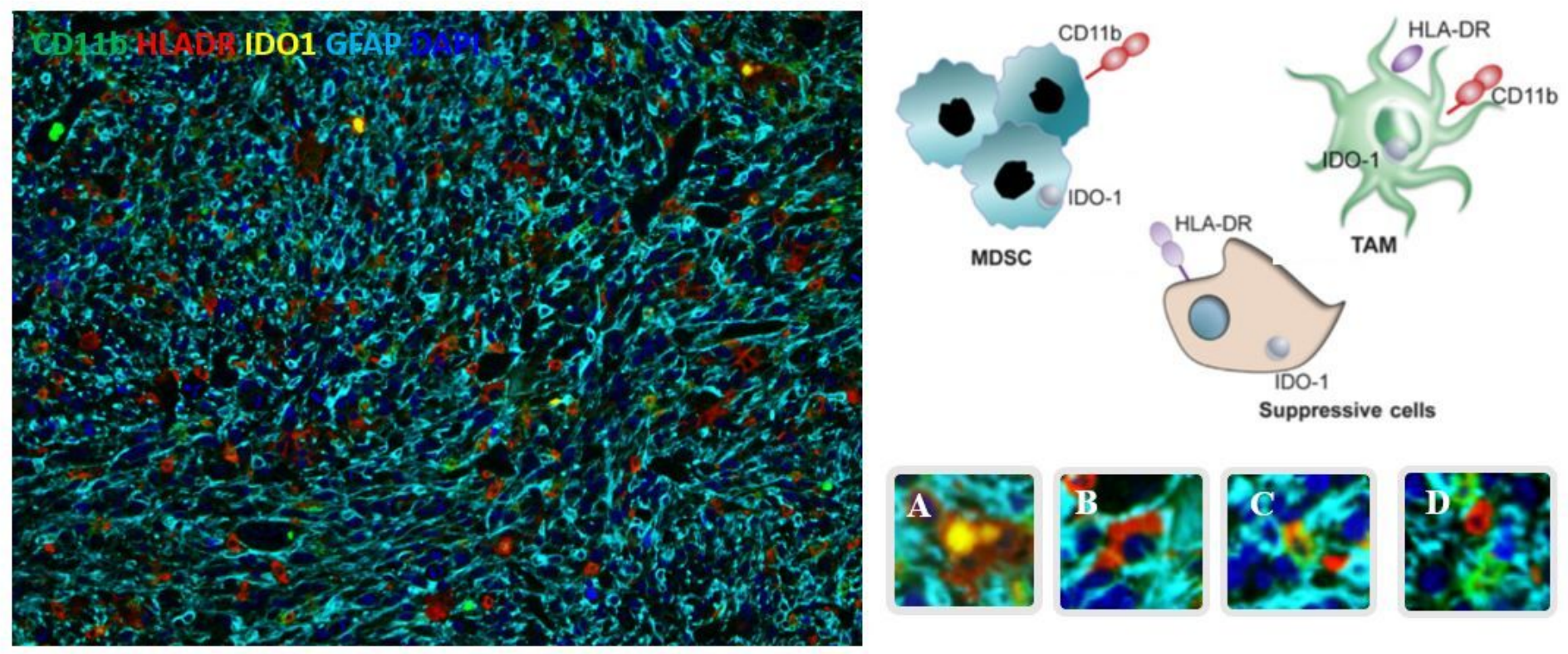

\section{Figure 2}

Myeloid-derived suppressor cell (MDSC) identification from biomarkers in GBM tissue. HLADR+IDO+ (A), HLADR+GFAP+ (B), CD11b+HLADR+ (C), CD11b+ (D)
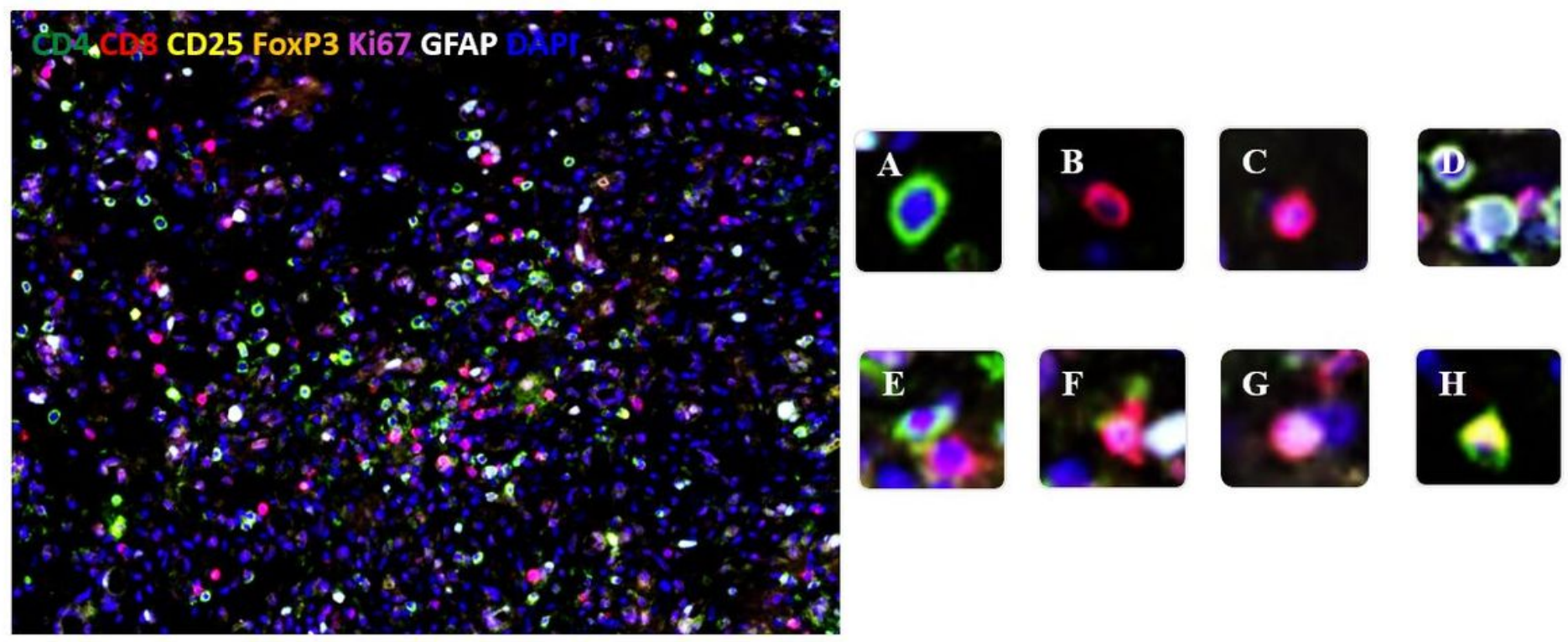

\section{Figure 3}

Enumeration of $T$ cells and their proliferation status from biomarkers in GBM tissue. CD4+ (A), CD8+ (B), $\mathrm{KI} 67+(\mathrm{C}), \mathrm{GFAP}+(\mathrm{D}), \mathrm{CD} 4+\mathrm{KI} 67$ (E), CD8+KI67+ (F), KI67+GFAP+ (G), CD4+CD25+FOXP3+ (H) 